\title{
Einsatz eines didaktisch gelenkten Chatrooms im Fremdsprachenunterricht
}

\author{
Lela-Rose Engler (Berlin)
}

\begin{abstract}
The use of virtual chatrooms for foreign language learning processes has lead to new research questions in the area of language acquisition. Assuming the differences between tutored and untutored chats tutored chats do not only offer the opportunity of synchronous communication between native and non-native speakers but also support the development of language abilities as reading and writing in the target language. In distance learning they can substitute for missing oral conversational classes.

The conditions which either do or do not support the use of chatrooms have to be taken into account for planning and evaluating distance foreign language classes.
\end{abstract}

\section{$1 \quad$ Wie kann ein Chat für den Fremdsprachenunterricht genutzt werden?}

Seit einigen Jahren werden didaktisch gestaltete Chaträume für den Fremdsprachenunterricht genutzt, um den Fremdsprachenerwerb zu unterstützen.

Neben E-Mail sowie Web- und Video-Konferenzen gehört die Chat-Kommunikation zu den zentralen Angeboten des Internets, die für fremdsprachliche Lernprozesse interessant erscheinen.

Für die fremdsprachendidaktische Praxis ergeben sich neben der Frage, welche didaktischen Möglichkeiten ein Chat bietet, noch eine Reihe anderer Aspekte. So zeigt die Analyse von im Fremdsprachenunterricht genutzten Chaträumen Merkmale und Besonderheiten, die in die Planung von Lernprozessen einbezogen werden müssen. Welche Fertigkeiten können durch das Chatten im Fremdsprachenunterricht gefördert werden? Können diese Fertigkeiten auch auf andere Kommunikationssituationen übertragen werden? Gibt es positive Einflüsse von Chat-Kommunikation auf den fremdsprachlichen Lernprozess?

Untersuchungen zum Einsatz von E-Mail und von Chaträumen im Fremdsprachenunterricht weisen auf den sog. "kommunikativen Mehrwert" hin, der zu einem Motivationsschub für den Erwerb einer Fremdsprache führt, da die Lernenden die Erfahrung machen können, dass in einem Chatraum tatsächlich mit der neuen, noch unvollkommen erworbenen Fremdsprache mit Sprechern der Zielsprache kommuniziert werden kann (vgl. Steinig et al. 1998). 
Neben den im Fremdsprachenunterricht bereits häufiger eingesetzten E-Mail- Korrespondenzen oder -partnerschaften, die nach Umfragen unter Fremdsprachenlehrenden in jeder Stufe einsetzbar sind, werden Chats hingegen eher mit fortgeschrittenen Lernern durchgeführt.

Dabei hat sich der Begriff "didaktischer Chatraum" = DCR, erstmals in der Lit. erwähnt von Steinig et al. (1998), als sehr hilfreich erwiesen. Dieser Chatraum muss sich von anderen Chats unterscheiden, wenn er zielgerichtet für die Unterstützung fremdsprachlicher Fertigkeiten eingesetzt werden soll. Es handelt sich dabei um einen Raum, der gezielt für Lerner/innen eingerichtet wird. Neben einer "Instanz", die den didaktischen Chatraum einrichtet und administriert und auch die Lehrer- bzw.Tutorrolle übernehmen kann, ist es die thematische Lenkung, auf der maßgeblich die kommunikative Tätigkeit im Chatraum basiert. So ist es sinnvoll, unter einer bestimmten sprachdidaktischen Zielstellung, den Chatraum nur für einen bestimmten Teilnehmerkreis zu öffnen ähnlich wie Teilnehmern eines Konversationskurses. Damit einher geht, dass die Teilnehmer in einem DCR nicht anonym agieren und üblicherweise in eine feste Unterrichts- und Gruppenstruktur eingebunden sind. Sprachliche Vorgaben durch Texte, Übungen, Vokabeln u. Ä. dienen als Vorbereitung auf die einzelnen Chatsitzungen und erfordern eine didaktische Lenkung durch den Tutor. Die Nutzung schriftlicher Sprache für die situationsgebundene, direkte und simultane Kommunikation ermöglicht unter fremdsprachendidaktischen Zielstellungen ein gezieltes Training an verschiedenen Sprachfertigkeiten. Durch die mediale schriftliche Realisierung der Kommunikation kann unter gesteuerten Spracherwerbsbedingungen das sprachliche Korrekturverhalten der Lerner stimuliert werden, da sie das Produzierte ständig nachlesen und überarbeiten können.

Auf mündliche Gesprächsstrukturen bezogen, können die Lerner/innen im Chatgeschehen verschiedene Gesprächsstrategien erwerben und trainieren, wie z. B. die Eröffnung eines Gespräches, die Beendigung, Themenwechsel u. a. Gleichzeitig werden Lese- und Schreibfertigkeit geübt. Hinzu kommt, dass der ständige Wechsel zwischen orientierendem und detailliertem Lesen, der beim Chatten gefordert wird, die Lerner/innen zwingt, das flexible Anwenden der unterschiedlichen Lesestrategien zu trainieren (vgl. Steinig et al. 1998).

Beispiele für den Einsatz von Chaträumen im Deutsch als Fremdspracheunterricht sind inzwischen zahlreich, genauere Analysen hingegen werden noch benötigt. Erfahrungen sind bisher für die Lernersprache Deutsch in dem 1998 durchgeführten Projekt von Steinig et al. (1998) beschrieben worden sowie in der Arbeit von Block (1999).

\section{Der Einsatz eines didaktischen Chatraumes in einem Fernkurs}

Im folgenden Beispiel soll es um den Chat in einem Distanzsprachkurs für Deutsch als Fremdsprache zwischen einer Berliner Universität und dem wirtschaftsjuristischen Fachbereich einer schwedischen Universität gehen, dessen Konzeption und Ziele von mir bereits in Linguistik online 9/2 (2001) dargestellt worden sind (vgl. Engler 2001). Dieser Kurs wurde zwischen 2000 und 2002 zweimal durchgeführt. Der Kurs selbst war als eine strukturierte HTML-Umgebung auf der Homepage des wirtschaftsjuristischen Instituts eingebunden. Zu diesem Distanzkurs fand kein begleitender Sprachunterricht vor Ort statt. Über die Kursseite gelangte man zu dem extra eingerichteten Chatraum, der die räumliche Distanz zwischen den 
Lernerinnen und der Tutorin überwinden sollte und den regelmäßigen virtuellen Treffen diente. Während der Chatsitzungen saßen die Teilnehmerinnen meistens an ihren heimischen Computern bzw. an räumlich getrennten Computerarbeitsplätzen auf dem Universitätscampus. Die Tutorin betreute den Kurs von Deutschland aus, sodass der Kontakt zwischen Tutorin und Teilnehmerinnen während des Kurses auf Emailaustausch und den Chatsitzungen basierte.

Bei den Zielgruppen handelte es sich in beiden Durchgängen um schwedische Muttersprachler im 1. Studienjahr Wirtschaftsrecht, von denen die meisten 5- 7 Jahre Deutsch in der Schule gelernt hatten, zwei Teilnehmerinnen aus dem 2. Jahrgang hatten lediglich 2 Jahre Deutsch in der Schule gelernt, dieses aber um längere Aufenthalte im deutschsprachigen Raum ergänzt. Das durchschnittliche Alter der Teilnehmerinnen lag bei 19 bis 21 Jahren, je eine Teilnehmerin aus den beiden Jahrgängen war durch vorherige Berufstätigkeit ca. 8 Jahre älter.

Der Chatraum, der eigens für die rtegelmäßigen Sitzungen eingerichtet und nur den Kursteilnehmerinnen zugänglich war, hatte sich als hervorragender Interaktionsraum für die beteiligten Teilnehmer im ersten Jahrgang erwiesen und war als sehr positiv und motivierend für die sprachliche Interaktion durch die Teilnehmerinnen beschrieben worden. Klar war jedoch auch geworden, dass die kommunikativen Aktivitäten der teilnehmenden Studenten nicht nur von technischen Schwierigkeiten beim Zugang zum Programm oder vom Thema der Diskussionen abhingen, sondern auch von der Gruppengröße. Deshalb war die Teilnehmergruppe des 2. Jahrganges in 3 kleine Gruppen eingeteilt worden, die idealerweise je 3 Teilnehmerinnen haben sollten. ${ }^{1}$ Häufig veränderte sich aber die Teilnehmerinnenzahl pro Gruppe besonders während der 2. Hälfte des Kurses. Das war u. a. dem Umstand geschuldet, dass es zum Wechsel der Unterrichtszeiten kam, was zu spontanen neuen Gruppenzusammensetzungen führte. Die Verkleinerung der jeweiligen Chatgruppe sollte der Dominanz eventuell kommunikativ stärkerer Teilnehmerinnen gegenüber kommunikativ schwächeren Teilnehmerinnen vorbeugen. Natürlich stellte auch die 3-stündige Chatzeit in der Fremdsprache im 1. Jahrgang eine enorme Anstrengung dar. So waren im 1. Jahrgang immer alle Teilnehmerinnen gleichzeitig im Chat, mit einer Chatzeit von insgesamt 3 Stunden, im 2. Jahrgang hingegen jeweils 3 Teilnehmerinnen für 1 Stunde.

Nicht überraschend ist natürlich, dass insgesamt 10 Chattreffen für die Teilnehmerinnen relativ wenige Gelegenheiten sind, in der Fremdsprache zu kommunizieren. Auch bekundeten die "neugierigen" Lernerinnen eine größere Freude an der Form des Chattens als eher traditionell orientierte Lernerinnen. Welche Chatdauer für welche Lernertypen günstig ist, gilt es weiterhin zu untersuchen. Meiner Beobachtung nach zeigten die starken Fremdsprachenlernerinnen auch nach 3 Stunden keine deutlichen Konzentrationsschwankungen, hingegen waren 3 Stunden Chat für schwächere Lernerinnen eine deutliche Überforderung. Eine einstündige Chatzeit hingegen stellte sich für die Schwächeren als relativ kurz heraus, da sie insgesamt viel

\footnotetext{
${ }^{1}$ Im ersten Jahrgang hatte die gesamte Gruppe zur gleichen Zeit fürjeweils ca. 3 Stunden gechattet. Im zweiten Jahrgang war die Gruppe in kleinere Gruppen geteilt worden. Die kleineren Gruppen waren jeweils ca. eine Stunde im Chat.
} 
Zeit für das Lesen und für das Schreiben aufwenden müssen. Dennoch wurden gerade die Chattreffen, die ja nur einen, wenn auch wesentlichen Bestandteil des Fernkurses ausmachten, positiv gewertet, wie einige Bemerkungen aus den abschließenden Befragungen zeigen. Interessant war hier neben der Einschätzung des Chattens als ein "Fast-Sprechen" oder als eine Kombination aus Sprechen und Schreiben durch die Teilnehmerinnen auch, dass das Fehlen des parasprachlichen und nonverbalen Bereiches der Kommunikation kein einziges Mal als Mangel benannt wurde, sondern im Gegenteil sogar als "gesichtswahrend" eingeschätzt wurde. ${ }^{2}$

B.: "Ich finde es sehr gut auf Deutsch zu chatten, auch wenn ich sicher viele verrückte sachen gesagt habe."

Mar.:" Ja, ich lerne viel wenn ich chatten. Das ist fast wie sprechen."

Mad.: "Gutes Kombination sprechen und schreiben."

Diese Aussagen zeigen, dass sich das Chatten für die Ausbildung fremdsprachlicher Fertigkeiten und Fähigkeiten vielseitig didaktisch nutzen lässt.

\section{$3 \quad$ Kriterien für die Analyse der Chatdaten}

Teilnehmer an Distanzkursen stehen vor einer besonderen Lernsituation: sie benötigen hohe Selfmanagementfähigkeiten, um ihren Lernprozess zu organisieren und eine hohe Motivation, da ein unmittelbarer "Ansprechpartner" fehlt: d. h. nicht jeder Lernertyp kommt mit der Fernlernsituation gut zurecht, unabhängig von äußeren Gegebenheiten wie ständigem Zugang zum Computer/Internet/Drucker etc. Geht es um Fremdsprachenunterricht, scheint es ein Paradox, kommunikative Fähigkeiten über einen Internetfernlernkurs zu erwerben, dem die wichtigen Sprachkomponenten des Hörens und des "klassischen Sprechens" fehlen. Wenn aber das Chatten als eine Mischung aus Sprechen und Schreiben betrachtet wird, dann lohnt es sich zu beobachten, welcher Modus, der eher schriftsprachliche oder der mündliche, in einem gelenkten fremdsprachlichen Chat verwendet wird. Handelt es sich um "verschriftlichte mündliche Sprache"? Eine erste Analyse hat ergeben, dass im vorliegenden Fall deutlich der schriftliche Modus überwiegt. Die der Technologie innewohnende Möglichkeit der Protokollierung des Chatgespräches kann positiv genutzt werden für den Fernlernfremdsprachenunterricht, wenn gezielt an korrekter Schreibung, korrektem grammatischen Gebrauch u. Ä. gearbeitet werden soll. So können auch schwächere Fremdsprachenlernerinnen rezeptiv vom Medium profitieren und dem Chatgeschehen in ihrem eigenen Tempo folgen, da es durch die schriftliche Protokollierung des Chatgeschehens ein ständiges "Nachlesen", Korrigieren oder "rewriting" erlaubt.

Damit können die ökonomischen Nachteile, die das Tippen im Chat gegenüber dem Sprechen mit sich bringt, durch die positiven Effekte der Schriftlichkeit kompensiert werden (vgl. Storrer 2001) und ermöglichen so ein differenziertes Arbeiten und eine gezielte Förderung schwächerer Fremdsprachenlerner/innen.

\footnotetext{
2 Interessant deshalb, weil die Chatteilnehmerinnen keine Philologiestudentinnen waren, die sich professionell mit den theoretischen Fragen des Spracherwerbs/des Sprachelernens beschäftigen.
} 
Ein grundlegender Unterschied zu sog. "freien" Chats bestand bei diesem Chat in seiner strukturellen und inhaltlichen Einbindung in einen Fernlernkurs und somit in dem Zwang, an dem Chat teilzunehmen und sich thematisch darauf vorzubereiten. Da es sich um vergleichbare Zielgruppen handelte, die mit den gleichen Themen arbeiteten, ist es möglich, sie miteinander zu vergleichen.

Im folgenden möchte ich mich auf zwei Themen, die die Grundlage zweier Chatsitzungen bildeten, konzentrieren. Diese Themen waren sowohl im 1. Jahrgang als auch im 2. Jahrgang jeweils bindend für eine Chatsitzung, die jeweils im 2. Teil des Fernkurses stattfand.

Unter vielen möglichen Kriterien für die Chatauswertung habe ich mich in diesem Beitrag für folgende entschieden: die Anzahl der Äußerungen der einzelnen Teilnehmerinnen (TN) im Verhältnis zu den Gesamtäußerungen im Chat, die Differenzierung der Äußerungen nach textthematischen Äußerungen und anderen (unterrichtsgestaltenden/organisatorischen/lernsituationsbedingten) und das Auftreten von Korrekturen.

Interessant ist es zu untersuchen, ob sich eine zunehmende Vertrautheit mit dem Medium anhand des Interaktionsverhaltens der Teilnehmerinnen beobachten lässt. Deshalb werden die kommunikativen Äußerungen in einem didaktischen Rahmen differenziert: ob sie themenbezogen oder nichtthemenbezogen sind. Denn wenn der Anteil der nichtthemengebundenen Äußerungen recht hoch ist, kann man m. E. davon ausgehen, dass das ein Indiz für ein generelles kommunikatives Verhalten in diesem Medium ist. Darüber hinaus ist für den Fremdsprachenunterricht besonders interessant, wie die Lerner "nichtthemengebundene" Situationen sprachlich meistern (die ja am ehesten "realen" Situationen im Zielsprachenland entsprechen), wie sie unabhängig von durch Texten vorgegebenen Sprachmustern ihr eigenes bereits vorhandenes Sprachwissen, Kontextwissen und auch ihr Welt- und Sachwissen aktivieren und sprachlich realisieren.

Unter themenbezogenen Äußerungen sollen in diesem Zusammenhang die Äußerungen verstanden werden, die sich auf die Textbearbeitung und Textdiskussion beziehen, die dem jeweiligen Chatthema zugrunde lagen. Alle anderen Äußerungen wie Begrüßung und Verabschiedung, organisatorische Fragen und Diskussionen, Fragen zu weiteren Elementen des Fernkurses werden als nichtthemenbezogen bezeichnet. Ein erwartbares Ergebnis, das ich an dieser Stelle vorwegnehmen möchte, konnte bestätigt werden: je nach Interesse am Thema schwankte natürlich auch die themenbezogene Äußerungsmenge einzelner Teilnehmer. Dennoch lässt sich beobachten, dass stärkere Lernerinnen, die sich auch in den Befragungen als positiv motiviert gegenüber der Nutzung eines Chatraumes äußerten, die stärkeren Chatteilnehmerinnen über alle Sitzungen hinweg bleiben. Interessant ist, dass geringes Interesse am Thema bei sprachlich schwächeren Fremdsprachenlernerinnen nicht so stark zu einer Abnahme der Äußerungshäufigkeit führte wie bei unmotivierten Teilnehmerinnen, die diese Art von Unterricht ablehnten. Sind die "stärkeren" Teilnehmerinnen nicht so sehr am Thema interessiert, so bleiben sie doch in der nichtthemagebundenen Kommunikation aktiv und halten ihren hohen Äußerungsanteil. Ähnliches kann auch für einige schwächere Lernerinnen beobachtet werden. Das aber wiederum scheint ein Indiz zu sein, dass die meisten Teilnehmerinnen den Chatraum durchaus als einen fremdsprachlichen Konversationsraum akzeptiert hatten und 
auch schwächere Sprachlernerinnen gerade von dem Medium profitieren, indem sie sich nach eigenen Aussagen im Chat weniger unter Druck gesetzt fühlen als in der mündlichen Konversationsklasse.

Was brachte die Veränderung der Gruppengröße und Chatzeit im 2. Jahrgang? ${ }^{3}$

Die Gruppen waren, wie bereits erwähnt, durch wechselnde Bedingungen instabil in Größe und Zusammensetzung. Trotz der meist geringeren Teilnehmerzahl im Chat während des 2 . Jahres wurden, relativ gesehen, mehr Äußerungen produziert. Das mag an der Gruppenzusammensetzung liegen, die allerdings willkürlich erfolgte bzw. an der Aufgeschlossenheit dem neuen Medium gegenüber und nicht zuletzt auch an dem Thema der Sitzung. Im Gegensatz zum 1. Jahrgang hatten einige Teilnehmerinnen des 2. Jahres allerdings schon einige Chaterfahrungen mit Englisch.

Die Anzahl der Äußerungen soll nach den o. g. Kriterien für 2 Sitzungen verglichen werden, die jeweils im 1. und im 2. Jahr stattfanden. Den Sitzungen lag der gleiche Text und das gleiche Chatthema mit den entsprechenden Leitfragen zugrunde und sollte neben der Vermittlung von landeskundlichen Kenntnissen und dem Vergleich zwischen Deutschland und Schweden zu einer kontroversen Diskussion anregen. Beide Themen wurden und werden bis heute auch in Deutschland sehr kontrovers diskutiert.

Sitzung zum Thema: Ladenschließzeiten

\section{Jahrgang 2000/2001}

Gesamtäußerungen der TN: 164 bei 3-stündiger Chatzeit (von 9- 12 Uhr)

\begin{tabular}{|l|l|l|l|}
\hline Name & Gesamtäußerungen & textthematische & $\begin{array}{l}\text { Korrekturen/Nachfra- } \\
\text { gen/Bemerkungen }\end{array}$ \\
\hline $\mathrm{T}$ & 61 Äußerungen & 34 Äußerungen & $\begin{array}{l}6 \text { Korrekturen (davon } 4 \\
\text { selbstinitiierte Selbst- } \\
\text { korrekturen) }\end{array}$ \\
\hline $\mathrm{Ch}$ & 40 Äußerungen & 24 Äußerungen & $\begin{array}{l}\text { ** (nimmt von den 3 Stunden für } \\
2 \text { Stunden am Chat teil) }\end{array}$ \\
\hline $\mathrm{R}$ & 34 Äußerungen & 15 Äußerungen & 1 Korrektur \\
\hline $\mathrm{S}$ & 18 Äußerungen & 9 Äußerungen & ------- \\
\hline $\mathrm{Ri}$ & 11 Äußerungen & 3 Äußerungen & ------- \\
\hline
\end{tabular}

S und Ri, auch im gesamten Fernkurs die schwächsten Lerner, haben in dieser Sitzung nicht nur die geringste Äußerungsanzahl, sowohl im textthemen- als auch nichttextthemengebundenen Kontext, sondern zeigen auch kein Korrekturverhalten. Die Teilnehmerin T dominiert deutlich, sowohl was die Äußerungsmenge betrifft als auch das Korrekturverhalten. Man kann vermuten, dass die Teilnehmerin Ch bei voller Anwesenheit im Chat zu einer ähnlich hohen Äußerungsmenge wie T gelangt wäre.

\footnotetext{
${ }^{3}$ Wie bereits erwähnt, war im Gegensatz zum 1. Jahrgang, wo immer die Ganzgruppe gemeinsam am Chat teilgenommen hatte, im " Jahrgang die Fernkursgruppe in 3 kleinere Chatgruppen geteilt worden.
} 


\section{Jahrgang 2001/02}

\section{Chatgruppe}

Gesamtäußerungen der TN: 110 bei ca. 1-stündiger Chatzeit

\begin{tabular}{|l|l|l|l|}
\hline Name & Gesamtäußerungen & textthematisch & $\begin{array}{l}\text { Korrekturen/Nachfra- } \\
\text { gen/Bemerkungen }\end{array}$ \\
\hline E. & 35 Äußerungen & 22 Äußerungen & \\
\hline J. & 18 Äußerungen & 12 Äußerungen & \\
\hline S. & 39 Äußerungen & 22 Äußerungen & $\begin{array}{l}2 \text { selbstinitiierte Selbst- } \\
\text { korrekturen }\end{array}$ \\
\hline Mad. & 18 Äußerungen & 13 Äußerungen & $\begin{array}{l}1 \text { selbstinitiierte Selbst- } \\
\text { korrektur }\end{array}$ \\
\hline
\end{tabular}

\section{Chatgruppe}

Gesamtäußerungen der TN: 87 (bei ca. 50 Min. Chatzeit)

\begin{tabular}{|l|l|l|l|}
\hline Name & Gesamtäußerungen & textthemat. Ä. & $\begin{array}{l}\text { Korrekturen/Nachfra- } \\
\text { gen/Bemerkungen }\end{array}$ \\
\hline B. & 53 Äußerungen & 30 Äußerungen & $\begin{array}{l}3 \text { selbstinitiierte. } \\
\text { Selbstkorrekturen }\end{array}$ \\
\hline Mar. & 34 Äußerungen & 20 Äußerungen & $\begin{array}{l}1 \text { selbstinitiierte Selbst- } \\
\text { korrektur }\end{array}$ \\
\hline
\end{tabular}

Im Gegensatz zum oben erklärten Ziel, die Gruppen des 2. Jahrganges gleichmäßig klein zu halten, nehmen an der Sitzung unterschiedlich große Chatgruppen teil: 4 Teilnehmerinnen von Chat 1 stehen 2 Teilnehmerinnen von Chat 2 gegenüber. Hinzu kommt, dass sich die Gruppen in dieser Sitzung anders gemischt hatten als in den vorangegangenen Sitzungen. Eine maßgebliche Ursache war dafür ein für die Teilnehmerinnen extremer Zeitwechsel. Statt des vormittäglichen Chats war die Chatzeit auf den späten Nachmittag gelegt worden, was dazu führte, dass nur 2 Chatgruppen für diese Sitzung gebildet wurden. Dennoch werden in den beiden Chats des 2. Jahrganges insgesamt wesentlich mehr Äußerungen produziert als in der Gruppe des 1. Jahrgangs, obwohl die Chatzeit jeweils eine gute Stunde kürzer ist. Beide Jahrgänge hatten das besprochene Thema als ausgesprochen interessant beschrieben. Was die Korrekturen betrifft, so gilt grundsätzlich, dass die Teilnehmerinnen mit den meisten Äußerungen auch am meisten korrigieren. 


\section{Jahrgang 2000/2001}

Gesamtäußerungen der TN: 172 (bei ca. 3-stündiger Chatzeit)

\begin{tabular}{|c|c|c|c|}
\hline Name & Gesamtäußerungen & textthematisch & $\begin{array}{l}\text { Korrekturen/Nachfra- } \\
\text { gen/Bemerkungen }\end{array}$ \\
\hline $\mathrm{T}$ & 80 Äußerungen & 43 Äußerungen & $\begin{array}{l}2 \text { selbstinitiierte Selbst- } \\
\text { korrekturen }\end{array}$ \\
\hline $\mathrm{Ch}$ & 36 Äußerungen & 25 Äußerungen & $\begin{array}{l}1 \text { selbstinitiierte Selbst- } \\
\text { korrektur }\end{array}$ \\
\hline $\mathrm{R}$ & 23 Äußerungen & 20 Äußerungen & \\
\hline$S$ & 1 Äußerung & 0 & \\
\hline $\mathrm{Ri}$ & 2 Äußerungen & 1 Äußerung & \\
\hline $\mathrm{E}$ & 30 Äußerungen & 28 Äußerungen & $\begin{array}{l}\text { (kam erst } 37 \text { min später } \\
\text { in den Chat) }\end{array}$ \\
\hline
\end{tabular}

Wie schon bei dem anderen Thema sind auch in dieser Chatsitzung $\mathrm{T}$ und Ch die äußerungsstärksten Teilnehmerinnen. Hinzu kommt noch E., die bei der anderen Sitzung (Thema Ladenschließzeiten) nicht teilnahm. T erreicht ihre sehr hohe Äußerungszahl u. a. dadurch, dass alle anderen Teilnehmerinnen später in den Chat kommen, sodass sie schon nichtthemengebunden sehr viel kommuniziert. Sie macht fast die Hälfte aller Äußerungen.

Die Unterschiede bei beiden Sitzungen bezüglich der allgemeinen Äußerungsmenge des 1 . Jahrganges sind darauf zurückzuführen, dass viel länger "allgemein" gechattet wurde bis zum eigentlichen Thema. In beiden Fällen fällt aber sofort die stärkste Teilnehmerin auf: T., die 61 Gesamtäußerungen zum 1. Thema aufweist und 80 zum 2.Thema.

\section{Jahrgang 2001/02}

\section{Chatgruppe:}

Gesamtäußerungen der TN: 60 (bei ca 1-stündiger Chatzeit)

\begin{tabular}{|l|l|l|l|}
\hline Name & Gesamtäußerungen & textthematisch & $\begin{array}{l}\text { Korrekturen/Nachfra- } \\
\text { gen/Bemerkungen }\end{array}$ \\
\hline S & 40 & 26 & $\begin{array}{l}\text { 4 selbstinitiierte Selbst- } \\
\text { korrekturen }\end{array}$ \\
\hline Mar & 20 & 9 & \\
\hline
\end{tabular}




\section{Chatgruppe}

Gesamtäußerungen der TN: 29 (bei 1-stündiger Chatzeit)

\begin{tabular}{|l|l|l|l|}
\hline Name & Gesamtäußerungen & textthemat. Ä. & $\begin{array}{l}\text { Korrekturen/Nach- } \\
\text { fragen/Bemerkungen }\end{array}$ \\
\hline $\mathrm{J}$ & 16 & 11 & \\
\hline $\mathrm{Ch}$ & 13 & 9 & \\
\hline
\end{tabular}

\section{Chatgruppe}

Gesamtäußerungen der TN: 133 (bei einer Chatzeit von 1h und 10 Minuten)

\begin{tabular}{|l|l|l|l|}
\hline Name & Gesamtäußerungen & textthemat. Ä. & $\begin{array}{l}\text { Korrekturen/Nachfra- } \\
\text { gen/Bemerkungen }\end{array}$ \\
\hline B & 62 & 31 & $\begin{array}{l}\text { 3 selbstinitiierte Selbst- } \\
\text { korr. }\end{array}$ \\
\hline Mad & 20 & 8 & $\begin{array}{l}\text { (kam eine Std. verspä- } \\
\text { tet) }\end{array}$ \\
\hline J B. & 28 & 21 & \\
\hline E & 23 & 21 & \\
\hline
\end{tabular}

Beim Thema "Kopftuchurteil" fällt besonders die 2. Chatgruppe des 2. Jahrganges auf. Hier scheinen die schon erwähnten veränderten Rahmenbedingungen (nur 2 Teilnehmerinnen, sie chatten sonst nicht in einer Gruppe, veränderte Unterrichtszeit, eine der beiden Teilnehmerinnen kritisiert diese Art von Unterricht) das kommunikative Geschehen wesentlich zu beeinflussen. Die Teilnehmerin J. steht dem gesamten Fernkurs kritisch gegenüber und ist trotz guter fremdsprachlicher Voraussetzungen nicht sehr kommunikativ - auch bei dem ersten Thema hat sie die geringste Äußerungsmenge. Das scheint auch das kommunikative Verhalten von $\mathrm{Ch}$ zu beeinflussen, die in anderer Gruppenzusammensetzung mehr Äußerungen produziert. Beide Teilnehmerinnen der Gruppe 2 haben eine Gesamtäußerungsmenge von 29, während bei gleicher Chatzeit und gleicher Teilnehmerinnenzahl in der 1. Gruppe die doppelte Anzahl von Äußerungen produziert wird und bei der 3. Gruppe mit doppelter Teilnehmerinnenzahl die Zahl der Äußerungen mehr als viermal so hoch ist. Das aber wiederum zeigt, wie auch im konventionellen Sprachunterricht, dass eine höhere Chatteilnehmerzahl als 3 nicht dazu führen muss, dass sich das gesamte Chatgeschehen verlangsamt.

Betrachtet man die Gesamtäußerungen für beide Themen und beide Jahrgänge unabhängig von den Gruppenzusammensetzungen des 2. Jahrganges, so fallen vor allem 2 Dinge auf. Die kleineren Gruppen des 2. Jahrganges produzieren mehr Äußerungen als die jeweilige Ganzgruppe des 1. Jahrganges. Besonders auffällig ist der Unterschied für Thema 1, da hier für den 2. Jahrgang die Chatzeit kürzer war.Weiterhin auffällig ist, dass es größere Abweichungen bei den nichtthemengebundenen Äußerungen im 1 Jahrgang gibt. Der Anteil dieser Äußerungen an der jeweiligen Gesamtäußerungsmenge im Chat liegt für den 1. Jahrgang bei $48 \%$ für 
Thema 1 und bei $32 \%$ für Thema 2. Dagegen ist die Abweichung für den 2. Jahrgang (39,6\% für Thema 1 und $38 \%$ für Thema 2) sehr geringfügig.

\begin{tabular}{|c|c|c|c|}
\hline Jahrgang & Thema & Äußerungen & Chatdauer \\
\hline $\begin{array}{l}\text { 1.Jgg. } \\
\text { eine Gruppe }\end{array}$ & Ladenschließzeiten & $\begin{array}{l}\text { - } \text { Gesamtäußerungen: } 164 \\
\text { - } \quad \text { Text-Äußerungen: } 104 \\
\text { - } \quad \text { andere Äußerungen: } 79\end{array}$ & 3 Stunden \\
\hline $\begin{array}{l}\text { 2.Jgg. } \\
\text { zwei Gruppen }\end{array}$ & Ladenschließzeiten & $\begin{array}{ll}\text { - } & \text { Gesamtäußerungen: } 197 \\
\text { - } & \text { Text-Äußerungen: } 119 \\
\text { - } & \text { andere Äußerungen: } 78\end{array}$ & 2 Stunden \\
\hline $\begin{array}{l}\text { 1.Jgg. } \\
1 \text { Gruppe }\end{array}$ & Kopftuchurteil & $\begin{array}{l}\text { - } \quad \text { Gesamtäußerungen: } 172 \\
\text { - } \quad \text { Text-Äußerungen: } 117 \\
\text { - } \quad \text { andere Äußerungen: } 55\end{array}$ & 3 Stunden \\
\hline $\begin{array}{l}\text { 2.Jgg. } \\
3 \text { Gruppen }\end{array}$ & Kopftuchurteil & $\begin{array}{l}\text { - } \quad \text { Gesamtäußerungen: } 222 \\
\text { - } \quad \text { Text-Äußerungen: } 136 \\
\text { - } \quad \text { andere Äußerungen: } 86\end{array}$ & $3 \mathrm{~h} 10 \mathrm{~min}$ \\
\hline
\end{tabular}

Wie bereits erwähnt, bietet das Medium durch die Möglichkeit der Protokollierung des Gespräches und des Nachlesens die Möglichkeit zur Korrektur. Das wird vor allem von den Chatteilnehmerinnen mit der höchsten Äußerungsmenge genutzt.

Die relative Höhe der nichtthemengebundenen Äußerungen lässt die Interpration zu, dass der Chatraum als eine Art fremdsprachlicher Konversationsraum verstanden wird, der durchaus Merkmale des konventionellen Klassenzimmers simuliert.

Besonders interessant beim Vergleich der beiden Jahrgänge ist die Tatsache, dass sich der Äußerungszuwachs im 2. Jahr syntaktisch messen lässt, da hier pro Äußerungseinheit wesentlich mehr Sätze gebildet werden als im 1. Jahrgang. Im ersten Jahrgang entspricht bei mehr als der Hälfte der Teilnehmerinnen die Zahl der Äußerungseinheiten auch der Zahl der Sätze. Im 2. Jahr sind wesentliche Unterschiede festzustellen. Jetzt werden pro Äußerungseinheit wesentlich mehr Sätze gebildet bei allen Teilnehmerinnen. Ob es sich dabei um einen Zufall handelt, ließe sich nur durch einen Vergleich mit weiteren Jahrgängen belegen, die an diesem Fernkurs unter gleichen Bedingungen teilnehmen.

Auffällig ist auch, dass bei beiden Jahrgängen das in verschiedenen Chatuntersuchungen beschriebene eher mündliche Muster der Chatsprache nicht zu beobachten ist. Es werden fast immer vollständige Sätze gebildet. Wo es nicht gelingt, liegt häufig eine technische Ursache zugrunde. Dass stärkere Fremdsprachenlernerinnen auch zu einem stärkeren Korrekturverhalten neigen, dürfte Fremdsprachenlehrer/innen nicht erstaunen, ebensowenig, dass hier die Art der selbstinitiierten Selbstkorrektur überwiegt, die durch die Schriftlichkeit des Mediums unterstützt wird . Dass stärkere Fremdsprachenlernerinnen auch in diesem Medium erfolgrei- 
cher sind, liegt nicht zuletzt daran, dass sie das fremdsprachliche Rezipieren und Produzieren einfach schneller bewältigen und in der Lage sind, die durch das Medium bereitgestellte Möglichkeit des "Nach"-Lesens viel besser für sich zu nutzen und in sprachlichen Strategien anzuwenden als schwächere Fremdsprachenlernerinnen.

\section{$4 \quad$ Einschätzung durch die Lernerinnen}

Wie schon bei dem 1. Jahrgang ist auch im 2. die Resonanz auf das Nutzen eines Chatraumes für den Fremdsprachenunterricht überwiegend positiv. Kritische Bemerkungen beziehen sich vor allem auf technische Schwierigkeiten. ${ }^{4}$ Störungen treten auch dann auf, wenn der gewohnte Unterrichtsrahmen durchbrochen wird. Zeitliche Verschiebungen von Chattreffen sowie sich verändernde Gruppenzusammen-setzungen können ein Hemmnis darstellen.

Als Vorteile des Chattens werden vor allem genannt:

- " man kann zu Hause arbeiten",

- " man hat das Gefühl, mehr im Chat zu reden als in der Direktkommunikation" ("weniger Scheu zu kommunizieren im Chat als im Face-to-face- Unterricht"),

- "Chatten ist eher Sprechen als Schreiben",

- $\quad$ man kann "Schreiben" üben.

Als Nachteile werden neben technischen Schwierigkeiten auch zu "schnelle" Diskussionen im Chatgeschehen, "bei denen man den Faden verliert", genannt. Erschwerend kam für 2 Teilnehmerinnen hinzu, dass sie über keinen eigenen Computer verfügten und deshalb ihren Computerzugang immer organisieren mussten. Bei der generellen Einschätzung dieser Art von Unterricht für den Fremdsprachenunterricht favorisieren die meisten Befragten einen Fernkurs mit den Schwerpunkten Chatten und Schreiben als eine Zusatzkomponente zu einem regulären Klassenkurs.

\section{$5 \quad$ Fazit}

Ein Chatraum, der im Sinne eines "Konversationsraumes" für den Fremdsprachenunterricht genutzt wird, kann den Face-to-face-Unterricht nicht ersetzen (und soll das auch gar nicht). Aber er ist eine große Bereicherung für die sprachliche Interaktion über die Distanz und kann bei sorgfältiger Planung der fremdsprachendidaktischen Lernziele durchaus die kommunikativen Fähigkeiten in der Fremdsprache trainieren und erweitern. Bei langfristigem Einsatz eröffnet sich die Möglichkeit, Gruppen nach unterschiedlichen Zielstellungen zu mischen, um das Dominieren eines besonders kommunikativen Mitgliedes einer Chatgruppe zu vermeiden oder auch passivere Teilnehmerinnen durch aktivere zu motivieren. Dabei sollte eine bestimmte Teilnehmerzahl nicht überschritten werden, nach meinen Erfahrungen bietet es sich an, mit 3 bis 4 Teilnehmerinnen gleichzeitig zu chatten.

Wenn allerdings die Chatgruppe aus 2 schwachen Fremdsprachenlernerinnen besteht oder eine Zweiergruppe eine unmotivierte Teilnehmerin hat, dann vermögen diese sich auch kaum

\footnotetext{
${ }^{4}$ Nur eine Teilnehmerin überhaupt spricht sich ausdrücklich gegen diese Art von Unterricht aus.
} 
zu motivieren und das Chatgeschehen verläuft stark tutoriell geleitet. Das bedeutete in diesem Fall, dass die Tutorin die passiven Teilnehmer konkret zu Äußerungen aufforderte und dass sich an diesen Stellen ein relativ monotones Gesprächsmuster beobachten lässt.

Diese Beobachtungen lassen sich mit anderen Unterrichtssituationen wie sie auch im mündlichen Konversationsunterricht auftreten, vergleichen. Gerade aber durch das Fehlen des parasprachlichen und nonverbalen Bereiches der Kommunikation im Chat wiegen sie umso schwerer und sollten bei ungünstiger Gruppenkonstellation durch ein stärkeres Intervenieren wie Nachfragen und direkte Aufforderungen zu Äußerungen seitens der Tutorin gemindert werden. Individuell auftretende Probleme können mittels einer sog. "private message", die nur für den Kurstutor sichtbar wird, signalisiert werden und können so verhindern, dass die offizielle Chatkommunikation gestört wird, indem sie parallel zur laufenden Diskussion ausgehandelt werden können. Das ist ein klarer Vorteil gegenüber der Klassensituation, wo die gesamte Gruppe durch eine Unterbrechung in einer Diskussion betroffen wird. Es erfordert lediglich vom Tutor/Kursleiter die Fähigkeit, parallele Kommunikationsstränge zu managen. Gegen Unmotiviertheit lässt sich m. E. im Chat genauso wenig (oder viel) tun wie auch im direkten Konversationsunterricht. Allerdings ist auch hier die sog. "Flüsterfunktion" in Form der "private messages" wiederum hilfreich für den Tutor, um inaktive Mitglieder der Chatgruppe anzusprechen. Da das wiederum für die anderen Teilnehmer nicht sichtbar ist, kann auch hier ein Gesichtsverlust vor der Gruppe vermieden werden. Für schüchterne Fremdsprachenlerner hingegen birgt die Kommunikation im Chatraum durchaus Vorteile neben den genannten. Neben der "Gesichtswahrung" können sie sich auf das Schriftliche wie z. B. auf grammatische, syntaktische und lexikalische Korrektheit konzentrieren.

Die Möglichkeit eines späteren Bezugnehmens auf zuvor (schriftlich) Realisiertes macht es im Gegensatz zur mündlichen Kommunikation möglich, dass "Gesagtes" für einige Zeit der Wahrnehmung der Kommunizierenden zur Verfügung steht. Durch die Ausschaltung des nonverbalen Kanals ist jegliche kommunikative Äußerung auf das schriftlich Geäußerte reduziert, was dazu führt, dass sich die Fremdsprachenlerner möglichst präzise und eindeutig in der Zielsprache äußern sollten, um den Fortbestand des Chatgespräches zu sichern. Das kann ihre Motivation erhöhen und letztendlich den Lerneffekt steigern. So sollten schriftliche Chatgespräche nicht aus der Perspektive der Defizite betrachtet werden gegenüber mündlichen Gesprächen, denn ihre medial schriftliche Realisierung kann die Nachteile der Unterbrechungen in der mündlichen Kommunikation durch das ständig nachlesbare Protokoll kompensieren. Verschränkungen, Subthemen und Überschneidungen in der fortlaufenden Chatkommunikation können wiederum das verstehende und sortierende Lesen in der Fremdsprache fördern. Chatprotokolle können sich in ihrer medialen Schriftlichkeit nicht nur während des Chatgespräches als vorteilhaft erweisen, sondern lassen sich auch in asynchrone Kommunikationsformen wie Email einbinden und für die Anschlusskommunikation oder z. B. dem gezielten Training im fremdsprachlichen Schreiben nutzen.

Ob primär mündliche oder schriftliche Sprachfertigkeiten in einem Distanzkurs trainiert werden, hängt natürlich von der Kurskonzeption ab. In diesem Fall wurden einige Chatthemen durch Schreibaufgaben, die sich auf das jeweilige Chatthema bezogen, ergänzt. Es bietet sich 
aber an, wie auch im traditionellen Sprachunterricht, die didaktischen Schwerpunktsetzungen $\mathrm{zu}$ variieren, um den unterschiedlichen Lernertypen besser gerecht zu werden. Schwieriger stellt sich hingegen die spontane Umstrukturierung einer Chatgruppe über die Distanz dar, wenn jeglicher Austausch und Kontakt zwischen Tutorin und Teilnehmerinnen über das schriftliche Medium erfolgt. Hier müssen die Lernerinnen den erforderlichen Organisationsaufwand selbst managen, was aber letztendlich ihre Autonomie fördert. Die Lernerinnen übernehmen also auch eine höhere Verantwortung für den eigenen Lernprozess.

Für mich überraschend war die Einschätzung durch die Teilnehmerinnen selbst. Fast alle akzeptieren den Chatraum trotz des Fehlens visueller und mündlicher/auditiver Unterstützung als einen Ersatz für den fehlenden klassischen Konverstaionsunterricht. Sie empfanden das Chatten als eine Mischung aus Sprechen und Schreiben mit einer starken Tendenz zum Sprechen. Und damit eröffnen sich ja unter didaktischer Perspektive genügend Lehr- und Lernmöglichkeiten für die Fremdsprache.

\section{Literaturangaben}

Block, Anja (1999): Online-Projekte im Fremdsprachenunterricht. Magisterarbeit, Universität Bielefeld. Online: www.uni-bielefeld.de/ ablock/texte/magister.htm.

Engler, Lela-Rose (2001): "Deutsch lernen über das Internet. Die Möglichkeiten eines didaktischen Chats". Linguistik online 9, 2/01.

Steinig, Wolfgang/Frederking, Volker/Berghoff, Matthias/Jünger, Werner (1998): "Fremde im Zug - Fremde im Netz: Ein interkulturelles Schreibprojekt". Zielsprache Deutsch 29, 1: 13-24.

Storrer, Angelika (2001): "Sprachliche Besonderheiten getippter Gespräche: Sprecherwechsel und sprachliches Zeigen in der Chat-Kommunikation". In: Beißwenger, M. (ed.): ChatKommunikation. Sprache, Interaktion, Sozialität \& Identität in synchroner computervermittelter Kommunikation. Perspektiven auf ein interdisziplinäres Forschungsfeld. Stuttgart. 